
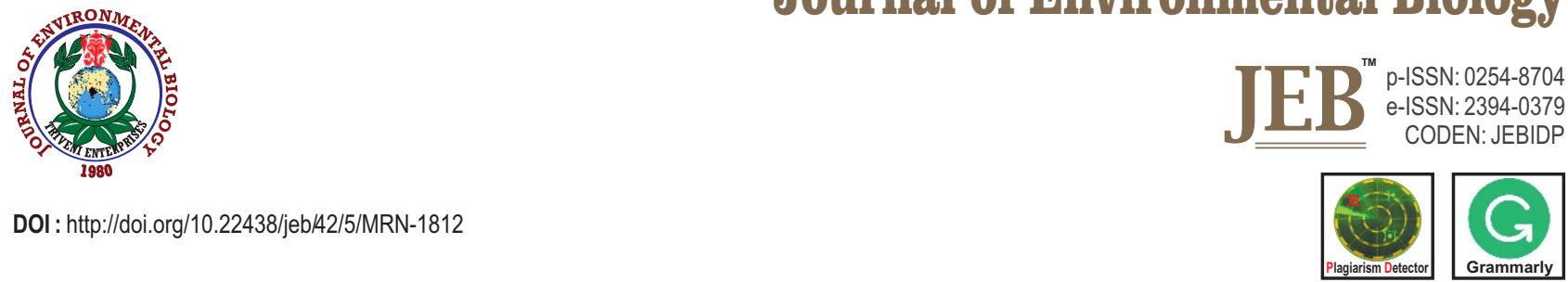

\title{
Development of tolerance to aldehyde-based inhibitors of pretreated lignocellulosic biomass sugars in $E$. coli MG1655 by sequential batch adaptive evolution
}

\author{
G. Padmapriya, V. Dhivya, M. Vishal, Y.A.J. Roshni, T. Akila and S. Ramalingam* \\ Centre for Biotechnology, Anna University, Chennai-600 025, India \\ *Corresponding Author Email : ramabioprocess@gmail.com
}

\begin{abstract}
Aim: The current study involved carrying out adaptive evolution to inculcate tolerance to hydrolysate-derived aldehyde-based inhibitors, furfural, vanillin, syringaldehyde and 4-hydroxybenzaldehyde (4-HB) for the valorization of pretreated lignocellulosic biomass.

Methodology: The growth-inhibitory effects of the aforementioned inhibitors on E. coliMG1655 were investigated. The percentage of inhibition was calculated from the initial growth, followed by extrapolating the $\mathrm{IC}_{50}$ values for each inhibitor. Based on these findings, adaptation experiments were conducted for individual inhibitors at a concentration lesser than or closer to $\mathrm{IC}_{50}$.

Results: The specific growth rate of cells was lowered by 2.2-, 3-, 1.3- and 5- fold when grown in the presence of furfural, vanillin, syringaldehyde and 4hydroxybenzaldehyde (4-HB), respectively. The adapted strains which were grown in the presence of furfural $(9 \mathrm{mM})$, vanillin $(9 \mathrm{mM})$, syringaldehyde (8mM) and 4- HB (6mM) individually showed around 1.5 -2.5- fold increase in the specific growth rate as compared to the wild-type with decreased lag phases and increased final cell densities.

Interpretation: The strains, subjected to adaptive evolution, resulted in increased tolerance to single inhibitors and these will further be sequentially adapted to other three inhibitors for their utilization in the valorization of pretreated lignocellulosic biomass.
\end{abstract}

Key words: Adaptive evolution, Aldehyde- based inhibitors, E. coli, Inhibitor tolerance, Specific growth rate

How to cite : Padmapriya, G., V. Dhivya, M. Vishal, Y.A.J. Roshni, T. Akila and S. Ramalingam: Development of tolerance to aldehyde-based inhibitors of pretreated lignocellulosic biomass sugars in E. coli MG1655 by sequential batch adaptive evolution. J. Environ. Biol., 42, 1239-1248 (2021). 


\section{Introduction}

The growing concerns of environmental pollution and the potential depletion of non-renewable sources have made scientists to discover sustainable microbial processes for the production of chemicals and fuels utilizing inexpensive and abundant feedstock (Namita et al., 2015). One such feedstock that fits the criteria is lignocellulosic biomass which is made up of tightly interlinked and intra-linked units of cellulose, hemicellulose and lignin as its major components. Several non-consumable materials such as bagasse, corn stover, wheat straw and rice husk, which are abundant, have also been used as a feedstock for microbial fermentation (Sánchez and Cardona, 2008; Balakrishnaraja et al., 2017). A significant drawback of using these resources as a microbial feedstock is mandatory requirement of pretreatment to overcome the inherent hydrolytic stability conferred by the recalcitrant materials, resulting in inevitable formation of degradation products such as furan aldehydes (furfural) and phenolic aldehydes (vanillin, 4hydroxybenzaldehyde and syringaldehyde) that are detrimental to microbial growth and productivity (Jönsson and Martín, 2016).

Though several detoxification methods have been reported, generation of toxic byproducts in case of harsh chemical detoxification and long incubation times, loss of sugars and comparatively higher costs of biological detoxification methods impede the practical usability of lignocellulosic biomass. Hence, there arose a need to explore targeted genome engineering and evolutionary adaptation strategies to develop a strain tolerant to inhibitors in the hydrolysate. Reports on genome engineering of microbes to impart inhibitor tolerance by overexpression of enzymes are sparse because of the difficulty in understanding the mechanism of stress tolerance in microbes (Liu et al., 2004). Random mutagenesis and genome shuffling are few methods which impart inhibitor resistance without the need to know the set of genes to be modified to effect beneficial mutations (Chandel et al., 2013; Bajwa et al., 2009). Some researchers suggest that the generation of inhibitor-tolerant strains using adaptive evolution has proved to be more successful than the above-mentioned genetic engineering strategies (Kurosawa et al., 2015).

Adaptive evolution of a strain imparts an improved inhibitor tolerance by iterative genetic diversifications and a process-relevant selection procedure in the presence of an inhibitor as selection pressure. Many process strategies for adaptation of cells in the presence of inhibitors have been reported, which include batch cultivations to achieve high cell densities, cell-retention cultivation using immobilized cells or cellrecirculation (Almeida et al., 2007). Long term adaptation experiments have been conducted either as a continuous chemostat system by maintaining very low dilution rates or in a sequential batch culture where cells would grow at $\mu_{\max }$ (Smith et al., 2014). Escherichia coli is one of the most widely used host strains in applied biotechnology for producing variety of microbial metabolites, including recombinant proteins (Waegeman and De,
2012), biofuels (Trinh and Srienc, 2009), and bio-products (Centeno et al., 2014). From industrial perspective, it offers several advantages over other commonly used organisms for bioprocesses, which include a very low doubling time of approximately one hour in defined media (Zaldivar et al., 1999), ability to ferment both hexose and pentose sugars, availability of extensive knowledge about the $E$. coli genome and regulation and extensive molecular tools (Idalia and Bernardo, 2017). Several protocols have already been established and optimized for different bio products with high turnover (Koppolu and Vasigala, 2016). These qualities make $E$. coli a lucrative platform for producing a versatile array of bio products from lignocellulosic biomass sugars cost-effectively.

In this study, E. coli MG1655 strains were developed individually tolerant to four most commonly found aldehydebased inhibitors in the hydrolysate: furfural, vanillin, syringaldehyde and 4- hydroxybenzaldehyde (4-HB). The concentration-dependent effect of these inhibitors on $E$. coli growth was assessed. Based on the inhibitory performance, strains with improved tolerance against the inhibitors were generated by the adaptive evolution approach. The aim of adaptive evolution is to improve the microbial growth rate, cell densities and shorten the lag phase in the presence of inhibitors for its use in pretreated biomass hydrolysates. Further, tolerance to other inhibitors may be made inherent by protoplast fusion or genome shuffling of strains tolerant to individual inhibitors or by subsequent adaptive evolution of these strains to other inhibitors. The objective of this study is to develop $E$. coli that is tolerant to the inhibitors in biomass hydrolysate, eventually resulting in efficient sugar utilization.

\section{Materials and Methods}

Microbial strain and culture media: The parent strain, E. coli MG1655 was maintained as glycerol stock in culture medium containing $25 \%$ glycerol at $-80^{\circ} \mathrm{C}$. The tolerance studies and evolution experiments were carried out at $37^{\circ} \mathrm{C}$ using $50 \mathrm{ml}$ of chemically defined minimal salts (M9) medium (Pardee et al., 1959), with $4 \mathrm{~g} \mathrm{I}^{-1}$ of glucose as carbon source, supplemented with inhibitors at various concentration, in $250 \mathrm{ml}$ flask. The salts were autoclaved separately while trace elements and inhibitors were filter sterilized using $0.2 \mu \mathrm{m}$ nylon filters. These sterile media components were mixed prior to inoculation. M9 medium without any inhibitors was used as a control for the study.

Tolerance studies in shake flask: To study the inhibitory effects of various inhibitors, $49.5 \mathrm{ml}$ of fresh M9 medium was aseptically added in a $250 \mathrm{ml}$ conical flask, followed by the addition of inhibitor at defined range of 2-10 mM for furfural, 2.5-12.5 $\mathrm{mM}$ for vanillin, 2-10 $\mathrm{mM}$ for syringaldehyde and 2-7 $\mathrm{mM}$ for 4hydroxybenzaldeyde. Each flask was inoculated with 1\% aliquots of overnight-grown culture. The flasks were incubated in an orbital shaker at $180 \mathrm{rpm}$ and $37^{\circ} \mathrm{C}$. The growth curves were determined by measuring the $\mathrm{OD}_{600 \mathrm{~mm}}$ of samples from each flask every hour. One unit of $O D_{600 \mathrm{~mm}}$ corresponds to $0.3 \mathrm{~g} \mathrm{l}^{-1}$ of dry cell 
weight of $E$. coli. The $\mathrm{IC}_{50}$ values for the inhibitors were calculated by plotting a graph between the percentages of inhibition, which was calculated by taking the $O D_{600 n m}$ values at the exponential phase of cell growth, versus the concentration of each inhibitor. A trend line was inserted to the graphs and the $\mathrm{IC}_{50}$ values of each inhibitor were taken as the concentration at which the percent inhibition was 50 .

Adaptive evolution of the wild type E. coli MG1655: A longterm evolutionary laboratory adaptation protocol for $E$. coli was developed using the $\mathrm{IC}_{50}$ values calculated from the previous observations to establish tolerance to inhibitors. To $50 \mathrm{ml}$ of M9 medium in a $250 \mathrm{ml}$ conical flask, $0.1 \%$ of overnight culture was aseptically inoculated. The initial sub-lethal concentration of each inhibitor was used and the flasks were kept in a shaker incubator at $37^{\circ} \mathrm{C}, 180 \mathrm{rpm}$. Passages were made periodically at every 24 -hr time interval by monitoring the cell growth. When the $\mathrm{OD}_{600 \mathrm{~nm}}$ of the sample was same or lesser than the previous passage, the cells were transferred iteratively to a medium containing same concentration of inhibitor. Once the strain had adapted to this concentration, it was then transferred to the medium containing a higher concentration of inhibitor and the same process was repeated till the strain got adapted to the desired concentration of inhibitor. After the adaptive evolution, the culture was streaked on M9 agar plates with inhibitors for isolated colonies. Glycerol stock cultures of adapted strains were prepared in culture medium with $25 \%$ glycerol and the adapted concentration of inhibitor and maintained at $-80^{\circ} \mathrm{C}$.

Evaluation of microbial adaptation in inhibitory media: To verify the effect of adaptation, comparative experiments were carried out on three independent populations. The adapted strain and wild-type strain were grown in $1 \%$ inoculum of M9 medium taken in $250 \mathrm{ml}$ conical flasks containing concentration of inhibitors that should be detrimental for the growth of wild-type strain. The native strain was inoculated without inhibitors and was used as control. The flasks were kept in a shaker incubator at $37^{\circ} \mathrm{C}, 180 \mathrm{rpm}$. Absorbance was read periodically every hour for 8 hrs. Time versus $\mathrm{OD}_{600 \mathrm{~nm}}$ was plotted to determine the effect of each inhibitor on the $E$. coliMG1655 strain.

Statistical analysis: The experiments were performed in duplicates and the results were expressed as mean. Standard deviations in these experiments varied between 0.001 and 0.042 .

\section{Results and Discussion}

To understand the effect of aldehyde-based inhibitors on various growth parameters, tolerance studies of $E$. coli MG1655 in the presence of vanillin, 4-HB, furfural and syringaldehyde individually were performed (Fig. 1). At first, the cells were grown in the presence of varying concentrations of vanillin, 4- HB,
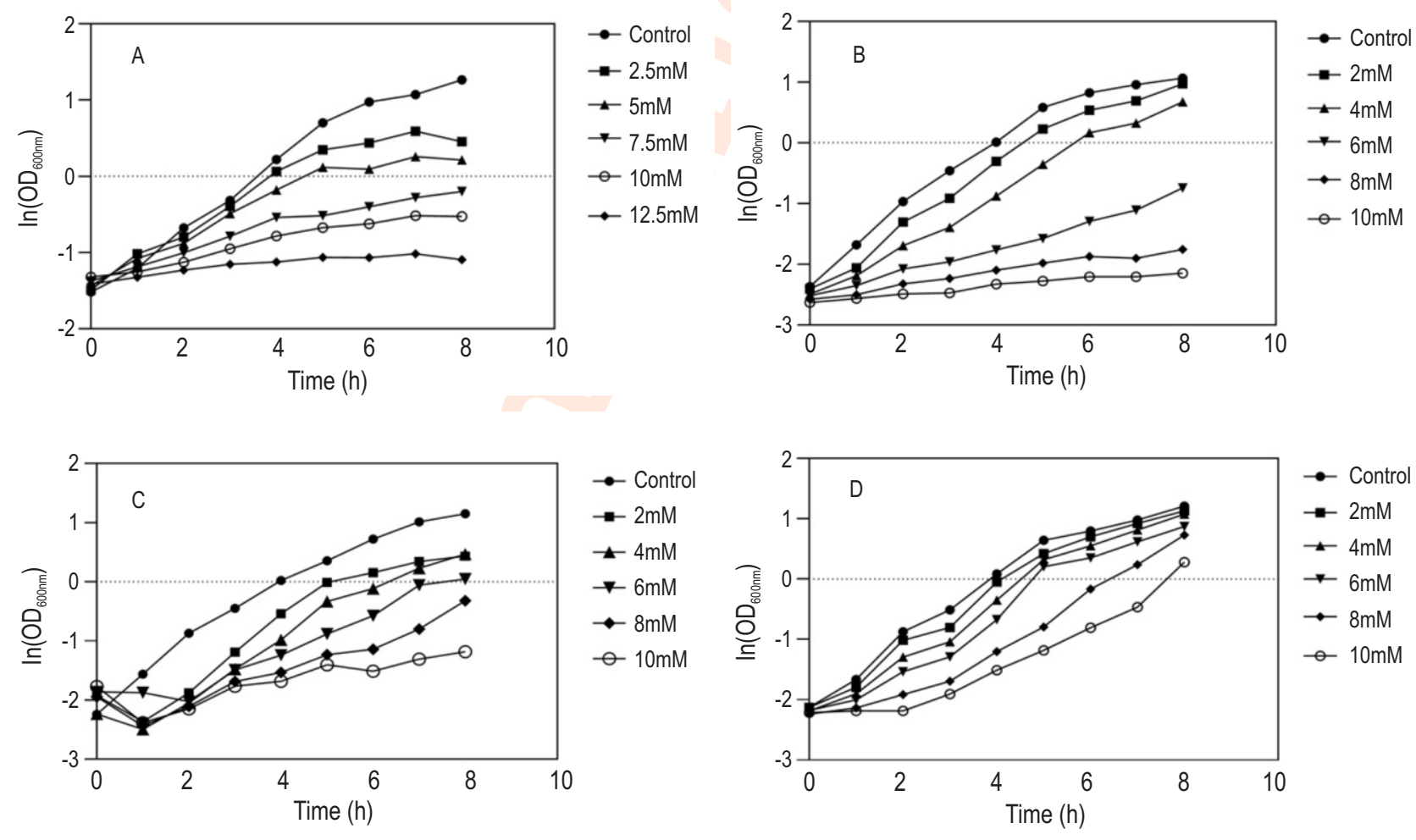

Fig. 1: Relationship between the growth of $E$. coli MG1655 and the concentration of (A) Vanillin; (B) 4-HB; (C) Furfural and (D) Syringaldehyde in the medium. (Standard deviation among the duplicates were below 0.008 ). 


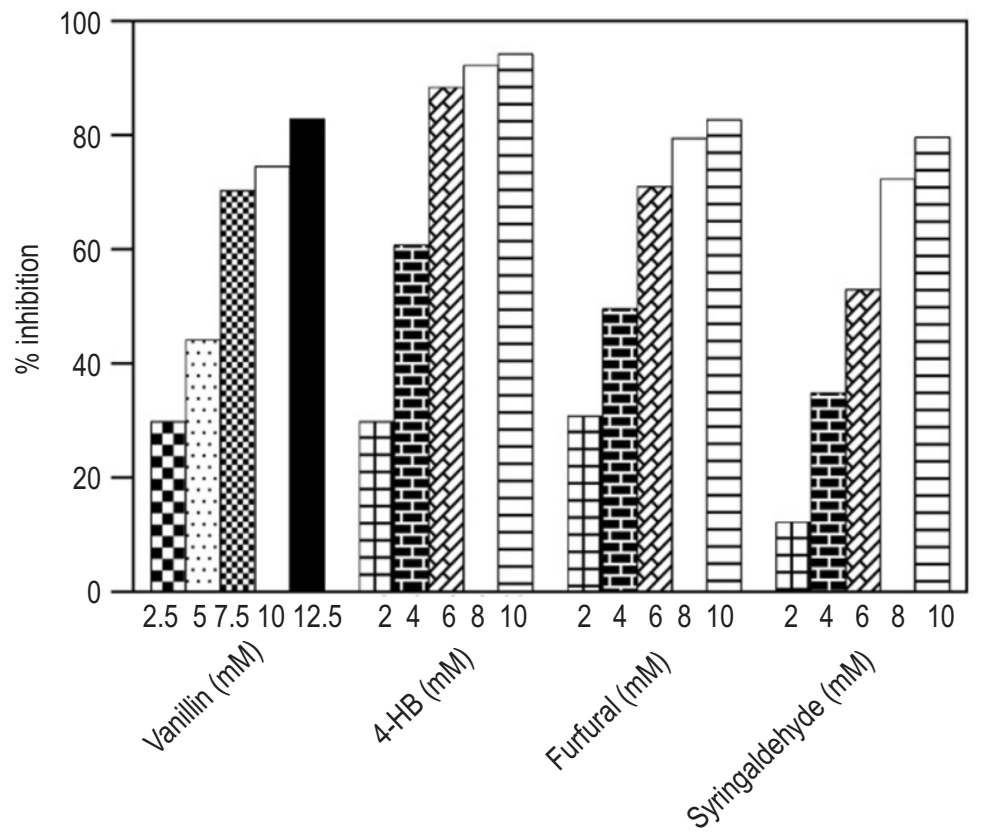

Fig. 2: Inhibitory effects of Vanillin, 4- HB, Furfural and Syringaldehyde on the growth of E. coli MG1655 (Standard deviation among the duplicates were below 0.005$)$.

furfural and syringaldehyde in the defined medium as stated in the materials and methods which was chosen based on the amount of inhibitors found in the biomass hydrolysates (Rumbold et al., 2009; Sannigrahi et al., 2010; Huang et al., 2011; Wilkinson et al., 2016; Du et al., 2010)along with control experiment without inhibitors. The amount of inoculum used in all these cultures was $0.1 \%$ and therefore no lag phase was found to exist in the control experiment and also in some of the cultures with inhibitors as well. The specific growth rates of all the cultures were monitored. In the control experiments, during early logarithmic phase, the cells grew exponentially and later started to decrease marginally either due to substrate depletion or product inhibition. In the case of vanillin and 4-HB, no lag phase was seen and the specific growth rate decreased with increasing concentration of inhibitors during the log phase of the culture. These results depict the influence of inhibitor concentration on the growth rate with almost no growth leading to complete inhibition for vanillin and 4-HB at 12.5 and $10 \mathrm{mM}$, respectively. Vanillin resulted in around 2.1- and 3- fold reduction in specific growth rates at 7.5 and $10 \mathrm{mM}$ concentrations, respectively, while, 4-HB resulted in 2.8- and 5- fold decline in specific growth rates at 6 and $8 \mathrm{mM}$ concentrations, respectively, as compared to the control experiment.

As far as furfural was concerned, in the lag phase, cell lysis was found to occur with decreasing cell densities and later the cells got adapted. Reports hypothesize that the adaptation process could take place during the extended lag phase before the recovery of active cell growth and metabolism (Liu et al., 2004). The specific growth rate decreased with increasing inhibitor concentration having 1.8- and 2.2- fold lower specific growth rates, at a furfural concentration of 6 and $8 \mathrm{mM}$, than that of the control experiment. In the case of syringaldehyde, no lag phase was seen at lower concentrations of inhibitors. However, with increasing concentration of inhibitor, the lag phase was observed for $2 \mathrm{hrs}$ indicating that cells took more time to enter the log phase. Further, during the early log phase, the specific growth rate was decreased with increasing concentration of inhibitor. During the mid-log phase, the specific growth of the cultures improved indicating that the adaptation took place and almost matched with that of the control experiment. Therefore, syringaldehyde resulted in only 1.3- fold reduction in the specific growth rate of cells at a higher concentration of $10 \mathrm{mM}$ when compared to that of control. Further, in the later phase, a decrease in growth due to the culture conditions as seen in the control experiment was observed. The reduction of both the growth rates and cell densities suggests that both the stoichiometry and the rate of conversion of substrates to cellular material were affected in the presence of inhibitors (Franden et al., 2013).

This decreased growth capacities of microbial cells, at higher inhibitor concentrations, could be connected to the expenditure of an increased fraction of total energy on cell maintenance in the presence of inhibitors (Zha et al., 2012). A similar observation was found in yeasts, where the addition of furfural resulted in altered intracellular $\mathrm{NAD}(\mathrm{P}) \mathrm{H}$ and ATP levels contributing to negative effects on energy generating glycolytic and TCA fluxes along with decreased activity/ inhibition of glycolytic enzymes and other dehydrogenases (Almeida et al., 2007, Sjulander and Kikas, 2020). Furan aldehydes (furfural) and phenolic aldehydes (vanillin, 4-HB and syringaldehyde) have 
A

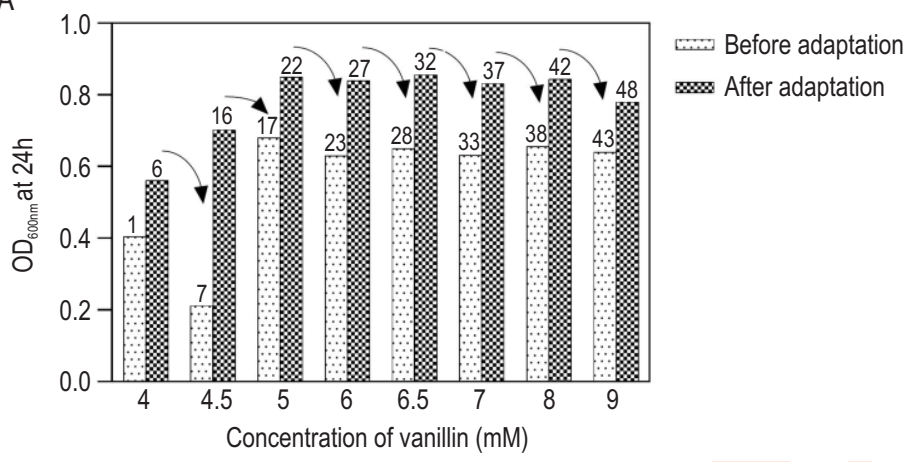

B

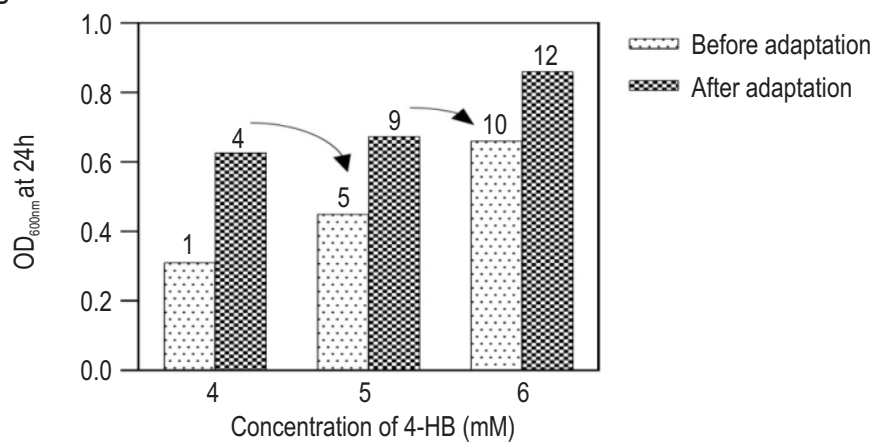

C

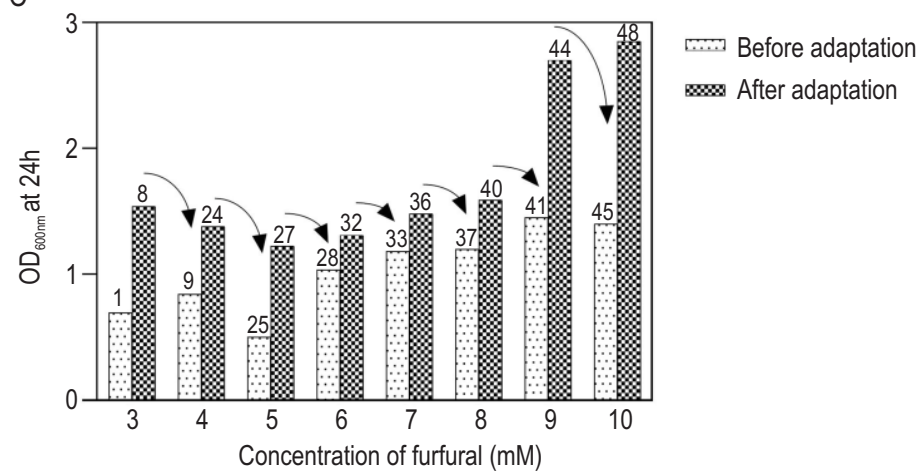

D

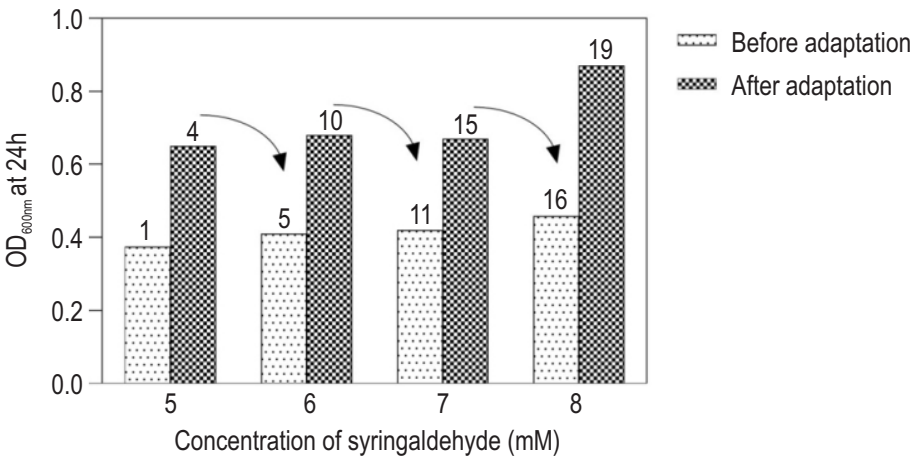

Fig. 3: Adaptive evolution of E. coli MG1655 in a defined medium containing a particular concentration of (A) Vanillin; (B) 4-HB; (C) Furfural and (D) Syringaldehyde. The number above the bar/ column indicates the number of passages or days of transfer and the arrow mark indicates transfer to a higher concentration of inhibitor (Standard deviation among the duplicates were between 0.003 and 0.042 ). 
been reported to hamper necessary metabolic processes by competing with the enzyme active site or depleting the nutrients or cofactors necessary for enzymatic activity (Mills et al., 2009). Based on the results obtained from the inhibitor studies, the percentage of inhibition exerted by each inhibitor at various concentrations was calculated (Fig. 2). This was further used to calculate the $\mathrm{IC}_{50}$ value of each inhibitor by graphical method. From the plot obtained, it was observed that growth kinetics does not linearly correlate with the inhibitor concentration, but rather becomes static at higher concentration. However, the points were fitted into a linear equation for two reasons: the extrapolated values were approximately equal to the actual values of growth $\left(R^{2}>0.95\right)$ and since the extrapolated values were slightly higher than the actual values, it would be more useful to adopt them for the adaptive evolution experiment. Therefore, with this slight approximation, the $\mathrm{IC}_{50}$ values of vanillin, 4- $\mathrm{HB}$, furfural and syringaldehyde were calculated to be $5.3 \mathrm{mM}, 3.4 \mathrm{mM}, 4.3 \mathrm{mM}$ and $5.7 \mathrm{mM}$, respectively. From the $\mathrm{IC}_{50}$ values, the growth inhibition of aldehydes on $E$. coli cells, listed in decreasing order of magnitude, was $4-\mathrm{HB}>$ furfural > vanillin > syringaldehyde.

The mechanism of inhibition of phenolic aldehydes has been reported to be the loss of membrane integrity by altering its protein to lipid ratio (Jönsson et al., 2013) and also affecting the other hydrophobic sites within the cell as well (Zaldivar et al., 1999). The toxicity of phenolic aldehydes in our study was also found to directly correlate to low molecular weight, orthosubstituent position and high hydrophobicity with the order of inhibition exerted by these phenolic aldehydes on E. coli MG1655 similar to that reported by Zaldivar et al. (1999). Based on the calculated $I_{50}$ value, the initial sub-lethal concentration of inhibitor for the adaptation process was found to be lower than or close to the $I \mathrm{C}_{50}$ for the respective inhibitor. The requirement at the end of the adaptation process was a strain with superior growth capacities having improved growth rates, higher final cell densities and lack of lag phase than the wild- type when grown in the presence of inhibitors. To conduct an efficient adaptation and selection regime for improved fitness of $E$. coli towards aldehydebased inhibitors, two key methods were adopted. Firstly, stepwise adaptation for a single inhibitor at a time was done since the adaptation of cells to a combination of inhibitors was reported to impose significant growth suppression (Kurosawa et al., 2015). Secondly, late log phase cells were exposed to a relatively higher concentration of inhibitors along with low inoculum size to ensure substantial selection pressure.

Additionally, the cells exposed to a prolonged stationary phase before serial transfer were reported to show rapid evolutionary adaptation. These factors were incorporated in the selection regime for establishing a dynamic environment for the microbial population to acquire selective advantage for survival (Jönsson et al., 2013; Dragosits and Mattanovich, 2013) thereby ensuring the dominance of cells which showed improved tolerance to inhibitor. Thus, the adaptation experiment was initiated by inoculating $0.1 \%$ of $E$. coli MG1655 individually, in the defined medium containing $4 \mathrm{mM}, 4 \mathrm{mM}, 3 \mathrm{mM}$ and $5 \mathrm{mM}$ of vanillin, 4- hydroxybenzaldehyde, furfural and syringaldehyde respectively, for adaptation (Fig. 3). The cells were sequentially adapted to a vanillin concentration of $9 \mathrm{mM}$ in 8 phases. In the initial phase, the cells took around 6 passages to adapt to the concentration of $4.5 \mathrm{mM}$. However, at higher concentrations, the cells gained improved fitness in around 5-6 days. The final cell density on day 48 was 1.9-fold higher than that of day 1 , and hence the adaptation was stopped at this concentration.

In the case of 4-HB and syringaldehyde, the cells were sequentially adapted to a final concentration of 6 and $8 \mathrm{mM}$ in 3 and 4 phases, respectively. In both cases, the tolerance was achieved in just 4-6 passages in each concentration. The adaptation process was terminated for the inhibitors, 4-HB and syringaldehyde, at day 12 and day 19 when 2.8- and 2.3- fold higher final cell densities than that on day 1 were obtained respectively. In the case of furfural, the cells were adapted to a final concentration of $10 \mathrm{mM}$ in 8 phases. In the initial phase, the cells struggled for 8 and 16 passages to get acquainted with the initial concentration of 3 and $4 \mathrm{mM}$, respectively. Further adaptation caused rapid shortening of lag phase, with 3-5 passages to get accustomed to a particular concentration of furfural and a significant increase in the cell densities after 40 passages. The final cell density at day 48 was 4 -fold higher than that of day 1 , and hence the adaptation experiment was terminated. In all the cases, the cells struggled for initial passages and later gained fitness to survive in the presence of inhibitor. Particularly, in the presence of furfural, growth rate started to improve only after 40 passages as opposed to syringaldehyde which required only limited passages to achieve a positive growth rate.

In addition to this, an increased lag phase with a decreased final cell- density was observed when the cells were grown in the presence of inhibitors, as compared to the control strain. The prolonged lag phase during the initial adaptation experiment might be attributed to the dominance of nonbeneficial mutations for survival where maximum cell adaptation process could occur (Smith et al., 2014). This is followed by increased beneficial mutations contributing to the synthesis of new enzymes and cofactors, and in doing so recovering its active cell metabolism (Huang et al., 2009) and growth by conversion of aldehydes to less toxic corresponding alcohols or acids (Boguta et al., 2014; Miller et al., 2009). Particularly, in the case of furfural, the increased flux through pentose phosphate pathway has been implied to be the most probable reason for the tolerance of cells to this inhibitor (Gorsich et al., 2006).

This observation also coincides with the reports which suggest that over-expression of enzymes like alcohol dehydrogenase, pentose phosphate pathway enzymes, phenyl acrylate decarboxylase and laccase have been reported to impart resistance to inhibitors in hydrolysate (Heer and Sauer, 2008; Kim and Hahn, 2013; Parawira and Tekere, 2011; Larsson et al., 2001). Over-expression of genes encoding reductase enzymes has also been reported to improve tolerance to 4hydroxybenzaldehyde and vanillin (Yang et al., 2018). It has been 
A

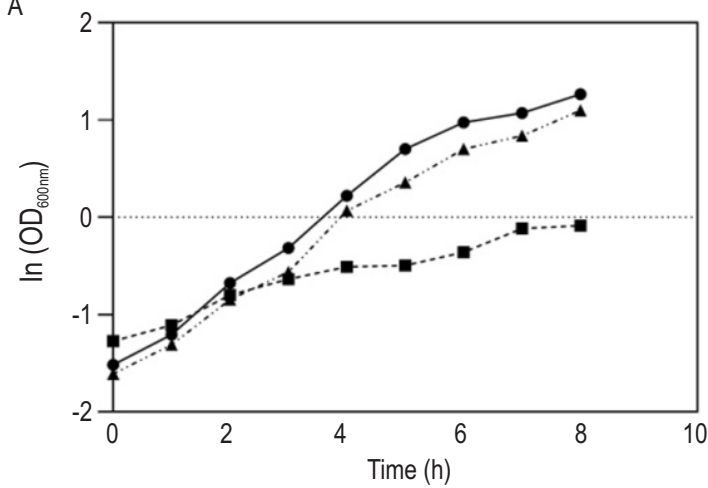

$\rightarrow$ Control

-- Wild-type

-. Adapted

B

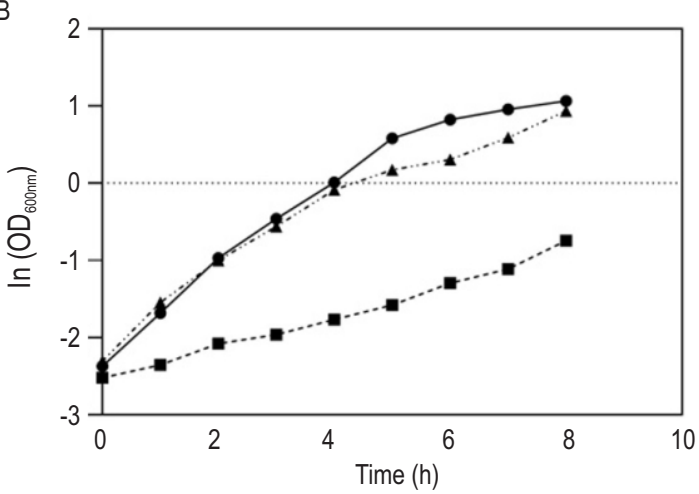

- Control

-.- Wild-type

«" Adapted

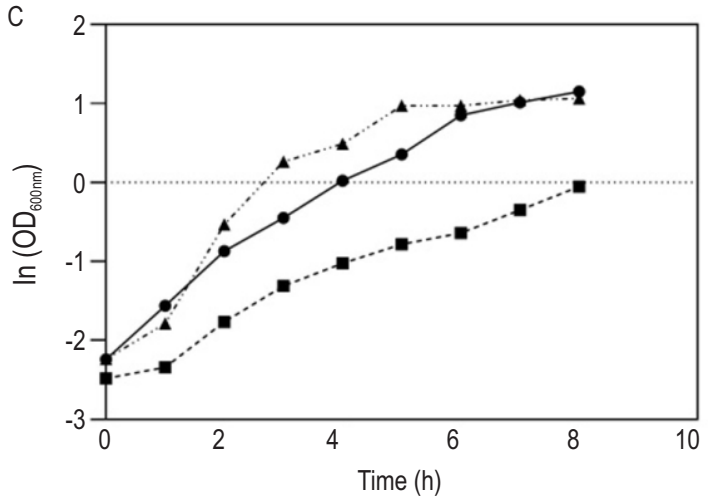

- Control

-* Wild-type

^. Adapted

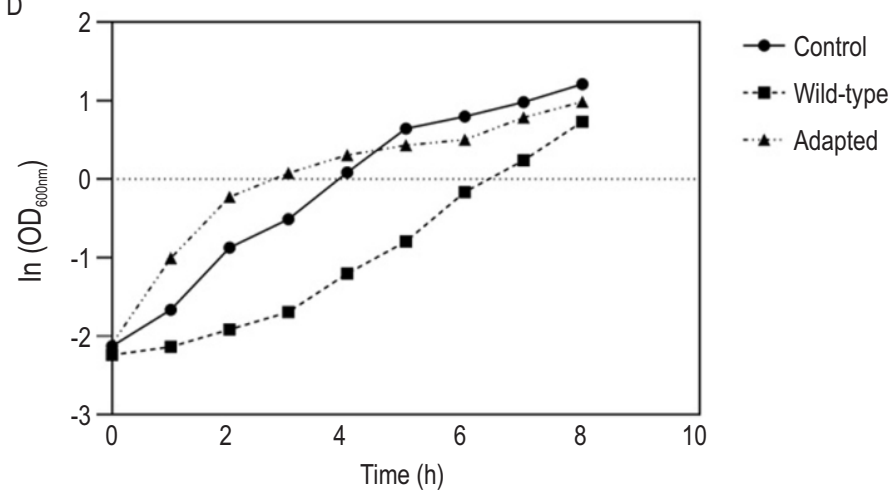

Fig. 4: Comparison of the growth of wild-type and adapted E. coli MG1655 in the presence of (A) 9 mM Vanillin; (B) 6 mM 4-Hydroxybenzaldehyde; (C) 9 $\mathrm{mM}$ Furfural and (D) $8 \mathrm{mM}$ syringaldehyde. E. coli MG1655 grown in media without any inhibitor was used as control (Standard deviations among the duplicates were between 0.001 and 0.007 ). 


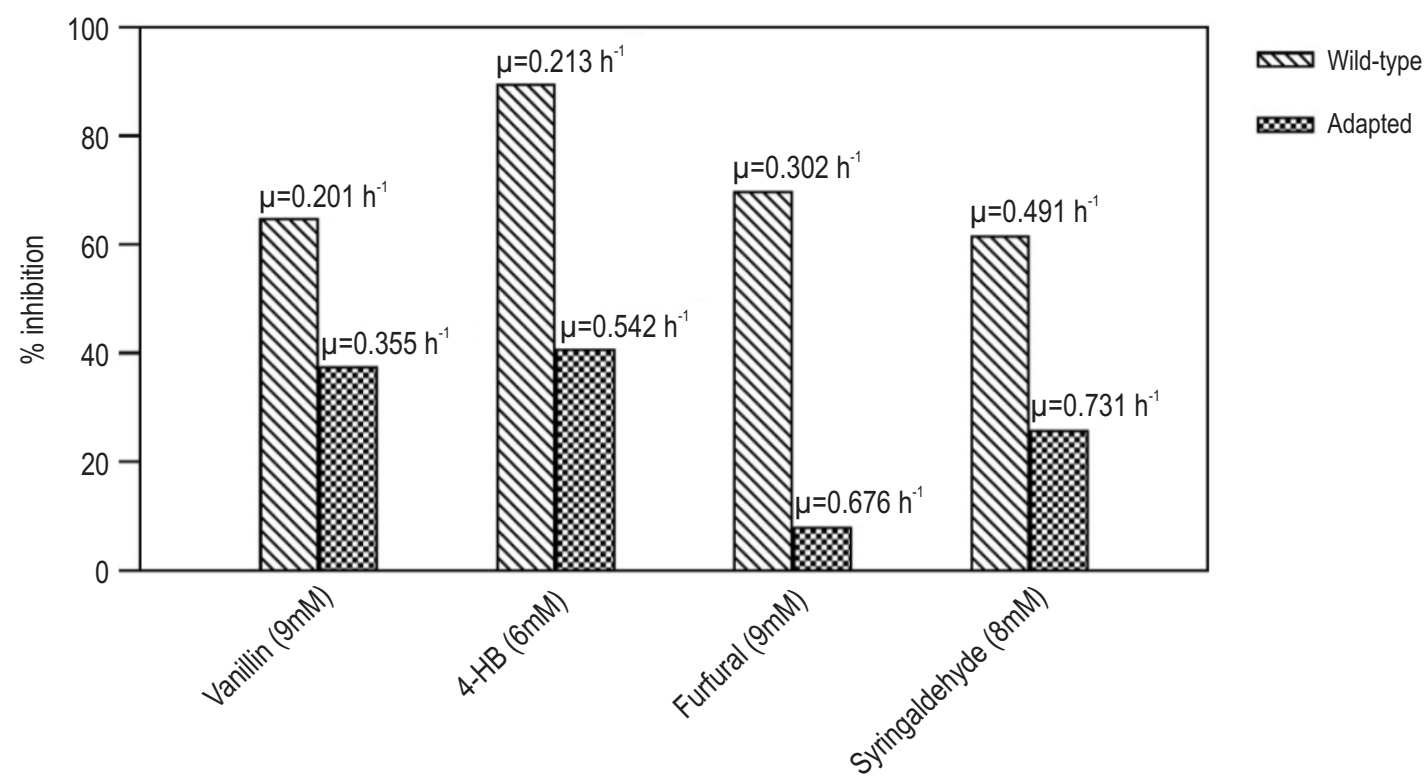

Fig. 5: Level of inhibition of Vanillin, 4- HB, Furfural and Syringaldehyde on the growth of adapted and wild-type strain. E. coli MG1655 grown in absence of inhibitors was used as control (Standard deviation among the duplicates were below 0.005 ). The value on top of each bar indicates the specific growth rate of that species in $h^{-1}$.

reported that adaptation using repeated batch process with replacement of fresh medium containing new load of inhibitors forced the cells to become tolerant owing to constant exposure of these cells to non-reduced form of inhibitors (Wallace and Gorwa, 2013). Further to evaluate the tolerance of developed strains, the adapted and wild-type $E$. coli were grown in the presence of final adapted concentration of vanillin, 4- HB, furfural and syringaldehyde, in a defined medium as stated in materials and methods along with the control, E. coli MG1655, grown in absence of an inhibitor. The amount of inoculum used in all these cultures was $1 \%$ and, in each case, the specific growth rates, length of lag phase and final cell densities achieved were monitored. Each adapted population displayed an increase in growth rate over the wild-type strain in the presence of an inhibitor (Fig. 4).

In the case of vanillin, 4- HB, furfural and syringaldehyde individually, no lag phase was observed with the adapted strains and they exhibited a 1.7-, 2.5-, 2.2- and 1.5-fold increase in the specific growth rates with a corresponding 3.3-, 5.4-, 3.1- and 1.3fold higher cell densities than the wild- type grown in the respective inhibitors. However, the final cell densities of adapted strain were lower than the control strain which could be considered as a trade-off event, for the utilization of low-cost pretreated lignocellulosic biomass (Wallace and Gorwa, 2013). Interestingly, specific growth rates of adapted strains in the presence of inhibitors were nearly close to that of the control experiment which shows the clear advantage of evolutionary adaptation resulting in a sugar utilization rate almost close to that of the cells grown using non- inhibitory carbon sources. In a similar study, fermentation of both glucose and xylose in the hydrolysate was faster for the adapted Saccharomyces cereviseae when compared to non-adapted one with higher ethanol specific and volumetric productivities (Martín et al., 2007). In contrast to traditional and molecular biological approaches, adaptive evolution improves inhibitor tolerance even in the absence of knowledge on the complex interaction of inhibitors with the biochemical network (Bajwa et al., 2010). The potential of adaptive evolution proves to be vital in improving inhibitor tolerance by allowing cell variants with selective advantage to exponentially take over the initial inhibitor- intolerant cell population. The estimation of percentage of inhibition exerted by the adapted concentration of inhibitors on the growth of adapted and wild- type $E$. coli was performed. The performance of adapted strains was exceptional with 1.7-, 2.2-, 8.7-and 2.4- fold decline in the percentage of inhibition exerted by vanillin $(9 \mathrm{mM}), 4-\mathrm{HB}$ $(6 \mathrm{mM})$, furfural $(9 \mathrm{mM})$ and syringaldehyde $(8 \mathrm{mM})$, respectively, when compared to wild- type E. coli (Fig. 5) proving further that the cells have got completely accustomed to the inhibitory environment (Kurosawa et al., 2015) and have gained fitness to be subjected for further adaptation to a combination of inhibitors thereby developing a tolerant E. coli MG1655 which could be efficiently utilized for the valorization of lignocellulosic biomass sugars. Studies suggest that one of the mechanisms which incorporate tolerance to inhibitors like furfural could be modulation of redox machinery (Jilani et al., 2020) or improved ability of MDR efflux pumps, porins, and other selected transporters in Escherichia coli (Kurgan et al., 2019).

Also, such single inhibitor tolerant strains have been reported to be necessary for the elucidation of mechanisms of 
stress tolerance (Liu et al., 2005). The development of inhibitor tolerant $E$. coli will have immense scope in industrial production of therapeutic proteins as well as value added chemicals by utilizing sugars in the lignocellulosic biomass. In this study, E. coli MG1655 individually tolerant to aldehyde-based inhibitors, furfural, vanillin, syringaldehyde and 4- HB have been developed which had improved specific growth rates and higher final cell densities as compared to the wild-type strain when grown in the presence of inhibitors. These strains, which were adapted to a single inhibitor, will further be subjected to adaptation to other three inhibitors for improved production of bio products by utilizing sugars from pretreated lignocellulosic biomass.

\section{Acknowledgments}

This study was funded by the Department of Biotechnology, India under the grant number, BT/EB/VC/01/2012 dated 22.09.2014. Partial grant of junior research fellowship from the Department of Biotechnology, Government of India and the Council for Scientific and Industrial Research, India are duly acknowledged.

\section{Add-on Information}

Authors' contribution: G. Padmapriya: Study conception, experimental design, conducted experiment, data collection, analysis and manuscript drafting; V. Dhivya: Conducted experiment, data collection and analysis; M. Vishal: Manuscript drafting; Y.A.J. Roshni: Conducted experiment and manuscript review; T. Akila: Manuscript review; S. Ramalingam: Study conception, experimental design and manuscript review.

Research content: The research content of manuscript is original and has not been published elsewhere.

Ethical approval: Not Applicable

Conflict of interest: The authors declare that they have no competing interests.

\section{Data from other sources: Not Applicable}

Consent to publish: All authors agree to publish the paper in Journal of Environmental Biology.

\section{References}

Almeida, J.R.M., T. Modig, A. Petersson, B. Hähn-Hägerdal, G. Lidén and M.F. Gorwa-Grauslund: Increased tolerance and conversion of inhibitors in lignocellulosic hydrolysates by Saccharomyces cerevisiae. J. Chem. Technol. Biotechnol., 82, 340-349 (2007).

Bajwa, P.K., D. Pinel, V.J.J. Martin, J.T. Trevors and H. Lee: Strain improvement of the pentose-fermenting yeast Pichia stipitis by genome shuffling. J. Microbiol. Methods., 81, 179-86 (2010).

Bajwa, P.K., T. Shireen, F. D’Aoust, D. Pinel, V.J.J. Martin, J.T. Trevors, and H. Lee: Mutants of the pentose-fermenting yeast Pichia stipitis with improved tolerance to inhibitors in hardwood spent sulfite liquor. Biotechnol. Bioeng., 104, 892-900 (2009).
Balakrishnaraja, R., S. Balasubramanian, P. Aravindan, A. Arulraj, K. Selvapriya, S. Geethadevi and R. Nisha: Optimization of ethanol productionusing pretreated corn cob and sugarcane bagasse hydrolysate by Candida parapsilosis strain BKR1. J. Environ. Biol., 38, 1357-1363 (2017).

Boguta, A. M., F. Bringel, J. Martinussen and P.R. Jensen: Screening of lactic acid bacteria for their potential as microbial cell factories for bioconversion of lignocellulosic feedstocks. Microb. Cell Fact., 13, 1-16(2014).

Centeno-Leija, S., G. Huerta-Beristain, M. Giles-Gómez, F. Bolivar, G. Gosset and A. Martinez: Improving poly-3-hydroxybutyrate production in Escherichia coli by combining the increase in the NADPH pool and acetyl-CoA availability. Anton. Van Leeuwen., 105, 687-696 (2014)

Chandel, A.K., S.S. Da Silva and O.V. Singh: Detoxification of lignocellulose Hydrolysates: Biochemical and metabolic engineering toward white biotechnology. Bioenergy Res., 6, 388-401 (2013).

Dragosits, M. and D. Mattanovich: Adaptive laboratory evolutionprinciples and applications for biotechnology. Microb. Cell Fact., 12, 1-17 (2013).

Du, B., L.N. Sharma, C. Becker, S.F. Chen, R.A. Mowery, G.P. Van Walsum and C.K. Chambliss: Effect of varying feedstockpretreatment chemistry combinations on the formation and accumulation of potentially inhibitory degradation products in biomass hydrolysates. Biotechnol. Bioeng., 107, 430-440 (2010).

Franden, M.A., H.M. Pilath, A. Mohagheghi, P.T. Pienkos and M. Zhang: Inhibition of growth of Zymomonas mobilis by model compounds found in lignocellulosic hydrolysates. Biotechnol. Biofuels., 6, 1 (2013).

Gorsich, S.W., B.S. Dien, N.N. Nichols, P.J. Slinger, Z.L. Liu and C.D. Skory: Tolerance to furfural-induced stress is associated with pentose phosphate pathway genes ZWF1, GND1, RPE1, and TKL1, in Saccharomyces cerevisiae. Appl. Microbiol. Biotechnol., $71,339-349$ (2006).

Heer, D. and U. Sauer: Identification of furfural as a key toxin in lignocellulosic hydrolysates and evolution of a tolerant yeast strain. Microb. Biotechnol., 1, 497-506 (2008).

Huang, C.F., T.H. Lin, G.L. Guo and W.S. Hwang: Enhanced ethanol production by fermentation of rice straw hydrolysate without detoxification using a newly adapted strain of Pichia stipitis. Bioresour. Technol., 100, 3914-3920 (2009).

Huang, H., X. Guo, D. Li, M. Liu, J. Wu and H. Ren: Identification of crucial yeast inhibitors in bio-ethanol and improvement of fermentation at high pH and high total solids. Bioresour. Technol., 102, 7486- 7493 (2011).

Idalia, V.M.N. and F. Bernardo: Escherichia coli as a model organism and its application in Biotechnology. In: Escherichia coli - Recent Advances on Physiology, Pathogenesis and Biotechnological Applications., DOI: 10.5772/67306 (2017).

Jilani, S.B., C. Dev, D. Eqbal, K. Jawed, R. Prasad and S.S. Yazdani: Deletion of pgi gene in $E$. coli increases tolerance to furfural and 5hydroxymethyl furfural in media containing glucosexylosemixture. Microb. Cell Fact., 19, 1-13 (2020).

Jönsson, L.J. and C. Martín: Pretreatment of lignocellulose: Formation of inhibitory by-products and strategies for minimizing their effects. Bioresour. Technol., 199, 103-112 (2016).

Jönsson, L.J., B. Alriksson and N.O. Nilvebrant: Bioconversion of lignocellulose: Inhibitors and detoxification. Biotechnol. Biofuels., 6,1-10(2013).

Kim, D. and J.S. Hahn: Roles of the Yap1 transcription factor and antioxidants in Saccharomyces cerevisiae's tolerance to furfural and 5-Hydroxymethylfurfural, which function as Thiol-Reactive 
electrophiles generating oxidative stress. Appl. Environ. Microbiol., 79, 5069-5077 (2013).

Koppolu, V. and V.K. Vasigala: Role of Escherichia coli in biofuel Production. Microbiol. Insi., 9, 29-35 (2016).

Kurgan, G., L.A. Panyon, Y.R. Sanchez, E. Pacheco, L.M. Nieves, R. Mann, D.R. Nielsen and X. Wang: Bioprospecting of native efflux pumps to enhance furfural tolerance in ethanologenic Escherichia coli. Appl Environ Microbiol., 85,1-11(2019).

Kurosawa, K., J. Laser and A.J. Sinskey: Tolerance and adaptive evolution of triacylglycerol-producing Rhodococcus opacus to lignocellulose-derived inhibitors. Biotechnol. Biofuels., 8, 1-14 (2015).

Larsson, S., P. Cassland and L.J. Jönsson: Development of a Saccharomyces cerevisiae strain with enhanced resistance to phenolic fermentation inhibitors in lignocellulose hydrolysates by heterologous expression of laccase. Appl. Environ. Microbiol., 67, 1163-1170 (2001).

Liu, Z.L., P.J. Slininger and S.W. Gorsich: Enhanced biotransformation of furfural and hydroxymethylfurfural by newly developed ethanologenic yeast strains. Appl. Biochem. Biotechnol., 121, $451-460$ (2005).

Liu, Z.L., P.J. Slininger, B.S. Dien, M.A. Berhow, C.P. Kurtzman and S.W. Gorsich: Adaptive response of yeasts to furfural and 5hydroxymethylfurfural and new chemical evidence for HMF conversion to 2,5-bis-hydroxymethylfuran. J. Ind. Microbiol. Biotechnol., 31, 345-352 (2004).

Martín, C., M. Marcet, O. Almazánand L.J. Jönsson: Adaptation of a recombinant xylose-utilizing Saccharomyces cerevisiae strain to a sugarcane bagasse hydrolysate with high content of fermentation inhibitors. Bioresour. Technol., 98, 1767-1773 (2007).

Miller, E.N., L.R. Jarboe, L.P. Yomano, S.W. York, K.T. Shanmugam and L.O. Ingram: Silencing of NADPH-dependent oxidoreductase genes (yqhD and $d \mathrm{kgA}$ ) in furfural-resistant ethanologenic Escherichia coli. Appl. Environ. Microbiol., 75, 4315-4323 (2009).

Mills, T.Y., N.R. Sandoval and R.T. Gill: Cellulosic hydrolysate toxicity and tolerance mechanisms in Escherichia coli. Biotechnol. Biofuels, 2, 1-11 (2009).

Namita, S., A. Devi, S. Kumar and A. Verma: Response surface methodology for standardisation of lignocellulosic biomass saccharification efficiency of NSF-2 fungus isolate. J. Environ. Biol., 36, 903-908 (2015).

Parawira, W. and M. Tekere: Biotechnological strategies to overcome inhibitors in lignocellulose hydrolysates for ethanol production: Review. Crit. Rev. Biotechnol., 31, 20-31 (2011).

Pardee, A.B., F. Jacob and J. Monod: The genetic control and cytoplasmic expression of "inducibility" in the synthesis of $\beta$ galactosidase in E. coli. J. Mol. Biol., 1, 165-178 (1959).

Rumbold, K., H.J.J. Van Buijsen, K.M. Overkamp, J.W. Van Groenestijn, P.J. Punt and M.J.V.D. Werf: Microbial production host selection for converting second-generation feedstocks into bioproducts. Microb. Cell Fact., 8, 1-11 (2009).

Sánchez, Ó.J. and C. A. Cardona: Trends in biotechnological production of fuel ethanol from different feedstocks. Bioresour. Technol., 99, 5270-5295 (2008).

Sannigrahi, P., Y. Pu and A. Ragauskas: Cellulosic biorefineriesunleashing lignin opportunities. Curr. Opin. Environ. Sustain., 2, 383-393 (2010).

Smith, J., E. Van Rensburg and J.F. Görgens: Simultaneously improving xylose fermentation and tolerance to lignocellulosic inhibitors through evolutionary engineering of recombinant Saccharomyces cerevisiae harboring xylose isomerase. BMC Biotechnol., 14, 1-17 (2014)

Sjulander, N. and T. Kikas: Origin, impact and control of lignocellulosic inhibitors in bioethanol production-A review. Energies, 13, 1-20 (2020).

Trinh, C.T. and F. Srienc: Metabolic engineering of Escherichia coli for efficient conversion of glycerol to ethanol. Appl. Environ. Microbiol., 75, 6696-6705 (2009).

Waegeman, $\mathrm{H}$. and M. De: Increasing recombinant protein production in E. coli by an alternative method to reduce acetate. Adv. Appl. Microbiol., 9, 1-11 (2012).

Wallace-Salinas, V. and M.F. Gorwa-Grauslund: Adaptive evolution of an industrial strain of Saccharomyces cerevisiae for combined tolerance to inhibitors and temperature. Biotechnol. Biofuels., 6 , 1-9(2013)

Wilkinson, S., D. Greethamand and G.A.Tucker: Evaluation of different lignocellulosic biomass pretreatments by phenotypic microarraybased metabolic analysis of fermenting yeast. Biofuel Res. J., 9 , 357-365 (2016).

Yang, Y., M. Hu, Y. Tang, B. Geng, M. Qiu, Q. He, S. Chen, X. Wang and $S$. Yang: Progress and perspective on lignocellulosic hydrolysate inhibitor tolerance improvement in Zymomonas mobilis. Bioresour. Bioprocess., 5, 1-12 (2018).

Zaldivar, J., A. Martinez and L.O. Ingram: Effect of selected aldehydes on the growth and fermentation of ethanologenic Escherichia coli. Biotechnol. Bioeng., 65, 24-33 (1999).

Zha, Y., B. Muilwijk, L. Coulier and P.J. Punt: Inhibitory compounds in lignocellulosic biomass hydrolysates during hydrolysate fermentation processes. J. Bioprocess. Biotechniq., 2, 1-12 (2012). 